\title{
LÍNEAS DE ACCIÓN Y PRINCIPIOS \\ PARA LA INCORPORACIÓN DEL APRENDIZAJE- SERVICIO EN EL ÁMBITO LOCAL. TRABAJO EN RED EN EL TERRITORIO
}

\author{
Lines of action and principles for the incorporation \\ of service-learning at local level. Networking in the territory
}

Brenda BÄR KWAST*; Laura CAMPO CANO** y Laura RUBIO SERRANO* *Universidad de Barcelona. España. brendabar@ub.edu; lrubio@ub.edu bttps://orcid.org/0000-0002-4263-4007; https://orcid.org/0000-0002-5170-4564 * Centro Promotor d'Aprenentatge Servei (Centro Promotor de Aprendizaje-Servicio). España.

centre@aprenentatgeservei.cat

Fecha de recepción: 15/07/2020

Fecha de aceptación: 21/09/2020

Fecha de publicación en línea: 01/11/2020

Cómo citar este artículo: Bär Kwast, B., Campo Cano, L. y Rubio Serrano, L. (2021 Líneas de acción y principios para la incorporación del aprendizaje-servicio en el ámbito local. Trabajo en red en el territorio. Teoría de la Educación. Revista Interuniversitaria, 33(1), 243-263. https://doi.org/10.14201/teri.23660

RESUMEN

El presente artículo tiene como objetivo aportar algunas reflexiones acerca de la potencialidad del aprendizaje-servicio desde un punto de vista territorial. La construcción de apuestas educativas en el ámbito local a través del aprendizaje-servicio permite superar el desarrollo de experiencias concretas y aisladas y avanzar hacia la construcción de redes educativas que tienen un horizonte de bien común. Los aportes 
BRENDA BÄR KWAST; LAURA CAMPO CANO Y LAURA RUBIO SERRANO LÍNEAS DE ACCIÓN Y PRINCIPIOS PARA LA INCORPORACIÓN DEL APRENDIZAJE-SERVICIO EN EL ÁMBITO LOCAL. TRABAJO EN RED EN EL TERRITORIO

en este sentido parten de una investigación experiencial de corte cualitativo que ha combinado el análisis documental de diferentes experiencias anteriores y el proceso de investigación-acción realizado en tres territorios catalanes (Barcelona, Sant Cugat y Puig-Reig). En este texto se exponen las principales líneas de acción a desarrollar desde la perspectiva de facilitación y/o coordinación en el proceso de implementar el aprendizaje-servicio a nivel territorial: aproximación al territorio, diagnóstico compartido; generación de proyectos; y comunicación de la apuesta territorial. La reflexión acerca de este proceso subraya que todas ellas se sustentan en base a principios como la apertura, el reconocimiento, la coherencia y la sostenibilidad, entre otros. Asimismo, el trabajo también apunta algunas reflexiones acerca de la agencia educativa por parte de otros agentes del territorio, más allá de los centros educativos, y el fortalecimiento del trabajo en red para generar territorios más educadores.

Palabras clave: aprendizaje-servicio; territorio; red educativa; comunidad; ciudad educadora.

\section{ABSTRACT}

This article aims to provide some reflections on the potential of service-learning from a territorial point of view. The construction of educational bets in the local scope through service-learning allows to overcome the development of concrete and isolated experiences and to advance towards the construction of educational networks that have a horizon of common good. The contributions in this sense are based on qualitative experiential research that has combined the documentary analysis of different previous experiences and the action-research process carried out in three Catalan territories (Barcelona, Sant Cugat and Puig-Reig). This text sets out the main lines of action to be developed from the perspective of facilitation and/or coordination in the process of implementing service-learning at a territorial level: approach to the territory, shared diagnosis; generation of projects; and communication of the territorial commitment. The reflection on this process underlines that all of them are based on principles such as openness, recognition, coherence and sustainability, among others. Likewise, the work also points out some reflections about the educational agency by other agents of the territory, beyond the educational centers, and the strengthening of the network work to generate more educational territories.

Key words: service-learning; territory; community; educational network; community; educating city.

\section{INTRODUCCIÓN}

Vivimos y convivimos en comunidad, nos organizamos y compartimos recursos que nos son comunes en ellas. Nos formamos como ciudadanos en comunidad y, seguramente también para ella; para que sea sostenible y nos permita vivir mejor: más libres y con el mayor bienestar posible para todas y todos. Con ello, no son 

EN EL ÁMBITO LOCAL. TRABAJO EN RED EN EL TERRITORIO

pocas ni desconocidas las dificultades y resistencias que esta vida en común supone. En un mundo donde el bienestar no está garantizado, al menos si lo pensamos como una realidad para todas las personas en su conjunto, es un imperativo ético indagar en formas de educar para una ciudadanía que sea capaz de caminar hacia su mejora. Educar para el compromiso con el entorno se vuelve indispensable ante una realidad social, económica y medioambiental que se presenta insostenible y que requiere cada vez más de una mirada interdisciplinar (Aznar y Barrón, 2017).

Existe una clara relación entre el aprendizaje-servicio y el desarrollo del compromiso social en sus participantes (Billig, Jesse \& Root, 2005). El aprendizajeservicio como propuesta educativa permite avanzar simultáneamente, y en constante retroalimentación, hacia una mejora educativa y una transformación social del entorno. Sus participantes aprenden de manera práctica y situada, a la vez que se implican en dar respuesta a necesidades sociales de manera altruista. Por todo ello, el aprendizaje-servicio, puede considerarse una práctica de ciudadanía (Puig, Gijón, Martín y Rubio, 2011).

En los últimos años, la propuesta de aprendizaje-servicio se ha extendido, tanto a nivel nacional como internacional, en diferentes ámbitos y niveles educativos que, van desde la escuela de primaria hasta la universidad, pasando por las organizaciones juveniles o su aplicación en instituciones que trabajan con jóvenes en situación de riesgo (Lorenzo, Santos Rego y Sotelino, 2015; Martín, 2018; Martínez, 2010; Naval, García, Puig y Santos, 2011; Puig, Batlle, Bosch y Palos, 2007; Puig, 2009; Rubio y Escofet, 2017; Tapia, 2001 y 2006). La potencialidad de la propuesta y el contexto educativo y social actuales han favorecido que muchas instituciones educativas, y cada vez un mayor número de entidades sociales, la conozcan e incorporen en su manera de trabajar.

Pero la capacidad transformadora del aprendizaje-servicio no reside simplemente en el valor de cada una de las experiencias tomadas de manera aislada. Es su despliegue en el ámbito local, y con concreciones diversas, lo que permite movilizar diferentes agentes del territorio y generar redes educativas locales que cristalizan el potencial educativo del barrio, el pueblo o la ciudad. De manera que se contribuye a construir una ciudad educadora (Trilla, 2005).

El contenido de este artículo se enmarca en la investigación y reflexión sobre las interacciones entre aprendizaje-servicio y territorio. En este marco, su aportación se concreta en algunas reflexiones y claves prácticas sobre dicha propuesta educativa en la construcción de un medio educativamente rico en el que el aprendizajeservicio, más allá de experiencias dispersas, avanza hacia su arraigo y consolidación local. Un proceso que requiere la facilitación y activación de mecanismos concretos desde el ámbito político, el técnico y directamente desde las y los responsables de la práctica educativa y social en el territorio. En este sentido, el estudio y seguimiento de diferentes experiencias en núcleos locales ha permitido llevar a cabo acciones de implementación, valorarlas con los diferentes agentes y extraer las conclusiones 
BRENDA BÄR KWAST; LAURA CAMPO CANO Y LAURA RUBIO SERRANO LÍNEAS DE ACCIÓN Y PRINCIPIOS PARA LA INCORPORACIÓN DEL APRENDIZAJE-SERVICIO EN EL ÁMBITO LOCAL. TRABAJO EN RED EN EL TERRITORIO

que se presentan en este artículo. Con el objetivo de presentar una sistematización de las principales líneas de acción y principios para poner en marcha un proceso en el que el aprendizaje-servicio se convierte en una práctica clave para construir comunidad y avanzar hacia el horizonte de bien común.

\section{EL APRENDIZAJE-SERVICIO COMO GENERADOR DE REDES LOCALES}

«El aprendizaje-servicio es una propuesta educativa que combina procesos de aprendizaje y servicio a la comunidad en una sola actividad bien articulada, donde los participantes se forman trabajando sobre necesidades reales del entorno con el objetivo de mejorarlo». (Puig, et al., 2007, p. 20). En el aprendizaje-servicio el desarrollo de una acción de servicio transforma y da sentido a los aprendizajes, permitiendo que sean prácticos y significativos, a la vez que éstos contribuyen a la mejora de la acción de solidaridad; combinando la intencionalidad pedagógica y la social.

Los antecedentes del aprendizaje-servicio pueden situarse en propuestas y autores que, desde la Escuela Nueva, destacaron el principio pedagógico de actividad (Martínez y Puig, 2011; Trilla, 2009). De esta manera, se reconocen los efectos educativos de una propuesta que, a través de la práctica, otorga un alto grado de protagonismo a sus participantes (Dewey, 1916; Freinet, 1971; Tonucci, 1979). Asimismo, se conoce el impacto formativo y transformador del voluntariado y las acciones solidarias en clave de comprensión crítica y formación de actitudes y valores (García Roca, 1994; Escámez, 2008; Martínez Odría, 2003). La novedad, en el aprendizaje-servicio se encuentra en integrar ambos elementos (servicio a la comunidad y aprendizaje significativo), ponerlos en interacción y, con ello, multiplicar sus efectos formativos.

Una de las características fundamentales del aprendizaje-servicio es que requiere de la colaboración entre agentes educativos y sociales, por lo que el partenariado se convierte en un elemento clave que le otorga calidad (National Youth Leadership Council, 2008; Service Learning Center de la Universidad de Stanford California, s.f.; Grup de Recerca d'Educació Moral [GREM], 2014). Las acciones se llevan a cabo con el territorio, recogiendo las necesidades de este, con un servicio que impacta en él y en el que se pueden conectar sus diversos agentes y entidades (Campo, López y Puig, 2013). El partenariado en el aprendizaje-servicio se entiende como colaboración entre dos o más instituciones educativas y sociales que posibilitan la realización conjunta de una actividad, contribuyendo positivamente a la consecución de sus objetivos y optimizando los recursos propios de unas y otras (Jacoby, 2003; Mulvihill, Hart, Northmore, Wolff \& Pratt, 2011). La puesta en marcha de este tipo de proyectos supone reconocer, conectar y trabajar conjuntamente entre centros educativos y entidades sociales que identifican necesidades y/o están interesadas en abordar su misión desde el trabajo con otros agentes (Sotelino, Mella y Rodríguez, 2019).

El partenariado puede tomar diferentes formas. De manera generalizada, los proyectos de aprendizaje-servicio delimitaban la aportación formativa al centro 

EN EL ÁMBITO LOCAL. TRABAJO EN RED EN EL TERRITORIO

educativo, mientras las entidades ofrecen un espacio de servicio a las y los participantes, se convierten básicamente en receptoras del mismo. Pero cada vez más, entidades y centros están generando nuevas interacciones y enriqueciendo las clásicas relaciones de partenariado. Las entidades también están explorando roles de colaboración, coordinación o impulso de los proyectos (Sotelino, et al., 2019). Existen experiencias en las que las entidades sociales ejercen su función como formadoras expertas o ejercen roles de coordinación entre diferentes entidades y agentes para enriquecer la acción. Con todo ello el aprendizaje-servicio contribuye a superar distancias excesivas entre las instituciones educativas y sociales, y avanzar hacia la idea de comunidad (Abravanel, 2003; Graell y Artaraz, 2016). Este es precisamente un primer paso para concretar lo que puede llegar a ser una red educativa.

Generar una red educativa requiere tomar una perspectiva local. En un contexto en el que todos los agentes y espacios son necesarios para educar con éxito resulta básico establecer entre ellos vínculos mutuos de corresponsabilidad y reciprocidad para construir proyectos educativos compartidos, que den respuesta a las necesidades formativas y conseguir los mejores resultados posibles (Collet y Subirats, 2016; Tort y Collet, 2017). Esta línea de trabajo parte de la idea de Ciudad Educadora que subraya la interrelación entre proyectos educativos que incorporan la visión de entorno y consideran la educación más allá de la escuela (Trilla, 2005) con acciones organizadas, potenciadas, coordinadas y al servicio de la ciudadanía. En definitiva, lo que algunos autores han apuntado como "territorializar" la educación y «educacionalizar» el territorio (Collet y Subirats, 2016). Son estas prácticas educativas de entorno, con carácter comunitario las que han de permitir superar el individualismo y generar comunidades fuertes basadas en su capital social (Díaz y Civís, 2011; Díaz, Civís y Longás, 2013; Sintes, 2015). Pese a ello, los estudios a nivel nacional e internacional señalan que todavía continúa siendo un reto encontrar experiencias arraigadas a redes locales y pensadas específicamente para generar una ciudadanía implicada en lo público (Díaz, et al., 2013).

Ante esta necesidad, y en un momento de revalorización de la innovación educativa que apunta el trabajo en red como un eje central, se desarrollan programas y proyectos que ponen el acento en los diferentes momentos, espacios y agentes educativos que han de fortalecer la educación (Sintes, 2015). Entre ellos, se debe reconocer la línea de trabajo que tanto a nivel nacional como internacional, queda enmarcada en la propuesta de Proyectos Educativos de Ciudad y Ciudades Educadoras (Asociació Internacional de Ciutats Educadores [AICE], 2009; Sánchez, Collet y Subirats; 2009; Institut de Govern i Politiques Publiques [IGOP], 2005; Trilla, 2005). A modo de ejemplo, en el ámbito catalán, el programa Educació 360/ Educació a Temps Complet ${ }^{1}$ apunta la idea del territorio como un espacio rico en

1. Alianza impulsada por la Diputación de Barcelona, la Federación de Movimientos de Renovación Pedagógica de Cataluña y la Fundación Bofill. Más en información en: https://www.educacio360.cat/ 
BRENDA BÄR KWAST; LAURA CAMPO CANO Y LAURA RUBIO SERRANO LÍNEAS DE ACCIÓN Y PRINCIPIOS PARA LA INCORPORACIÓN DEL APRENDIZAJE-SERVICIO EN EL ÁMBITO LOCAL. TRABAJO EN RED EN EL TERRITORIO

aprendizajes para cada persona, remarcando la necesidad de explorar el potencial de los ecosistemas educativos locales (Hannon, Thomas, Ward \& Beresford, 2019). En ambos casos, se trata de iniciativas que se caracterizan por reconocer los actores educativos más allá de la escuela y la necesidad de tejer redes de colaboración entre los diferentes agentes implicados en un territorio buscando una mayor coherencia y equidad en educación.

En este contexto, el aprendizaje-servicio representa una pieza clave y eficaz para generar, fortalecer y/o consolidar el trabajo en red en el ámbito local dado su componente territorial intrínseco. Los principios pedagógicos del aprendizajeservicio, su vinculación a necesidades reales y su intención de transformación social, hace que necesite de una mirada al entorno para poder ser, lo que la convierte en una propuesta directamente conectada con el territorio (Maroto, 2018). Han sido estudiados los impactos del aprendizaje-servicio en relación a diferentes ámbitos y niveles: a) en sus participantes y la formación educativa, cívica y de crecimiento personal (RMC Research Corporation, 2007; Roldan, Strange \& David, 2004; Winterbottom, Lake, Ethridge, Kelly \& Stubblefield; 2013); b) en los centros educativos, con la mejora de su convivencia y reducción de conflictividad (BIDCE, 2016, Gordó, 2018); c) en las comunidades que reciben el servicio (Balciuniene \& Mazeikiene, 2008; Harrington, 2014).

Por todo ello, y especialmente esto último, el aprendizaje-servicio es una propuesta pedagógica interesante para pueblos y ciudades, porque invita a conectar las instituciones educativas con el territorio, con sus necesidades, sus oportunidades de aprendizaje y posibilidades de acción en forma de servicio. Más allá de la proliferación de experiencias de aprendizaje-servicio concretas y ricas en partenariado, estas se erigen cada vez más como prácticas que pueden contribuir de manera decisiva en la construcción de apuestas educativas de entorno.

A pesar de no existir referentes internacionales claros acerca del abordaje del aprendizaje-servicio a nivel territorial, en el estado español hace ya un tiempo que son diferentes los municipios que están generando condiciones para avanzar en este sentido. De esta manera, cabe destacar la apuesta que desde algunas administraciones se ha hecho con relación al aprendizaje-servicio desde esta perspectiva. En el marco de la Red Española de Ciudades Educadoras (RECE) y en colaboración con la Red Española de Aprendizaje-Servicio ${ }^{2}$ se han recopilado diferentes experiencias que algunos ayuntamientos están realizando para luchar contra del fracaso escolar y mejorar la cohesión social del territorio a través del impulso del aprendizaje-servicio (Batlle, 2018a).

A nivel catalán, la implementación territorial del aprendizaje-servicio tuvo un impulso importante por primera vez a través de experiencias piloto en el marco

2. Los principales aportes en este sentido están recogidos en la página web de la Red Española de Aprendizaje Servicio: https://www.aprendizajeservicio.net/municipios-X-el-aps-2/ 
de los Planes Educativos de Entorno de la Generalitat de Cataluña en colaboración con el Centro Promotor de Aprendizaje-Servicio (Puig, 2010). Desde entonces, han sido diversos los municipios que se han interesado por el aprendizaje-servicio y, en este momento, se ha ampliado el número de instituciones y administraciones que lo impulsan. ${ }^{3}$ En este contexto cabe destacar el impacto que está teniendo el Decreto de Servicio Comunitario del Departamento de Educación (Generalitat de Catalunya, 2015), que ha supuesto, desde el curso 2019-2020, la incorporación obligatoria del aprendizaje-servicio como metodología de proyectos con vocación de servicio en los cursos de tercero o cuarto de la Educación Secundaria Obligatoria. Por todo ello, el Centro Promotor de Aprendizaje-Servicio y el Grupo de Investigación en Educación Moral (GREM) de la Universidad de Barcelona han trabajado en un documento que recoge algunas consideraciones que pueden orientar este proceso (Bär, Calvet, Campo, Puig y Rubio, 2020, en prensa).

Tal y como se apuntaba al inicio, incorporar el aprendizaje-servicio desde la perspectiva territorial requiere de un apoyo específico que active este proceso en cada municipio o ciudad (Puig y Campo, 2012). Para ello pueden aprovecharse las aportaciones de la planificación estratégica (Marchioni, 2001; Rueda, 1993) que apuntan una propuesta organizativa para estructurar los procesos, pero desde un enfoque flexible y adaptado a las diferentes realidades. Por su parte, el modelo de acción comunitaria (Barbero y Cortés, 2005; Carmona y Rebollo, 2009; Úcar y Llena, 2006) también resulta útil para observar cómo se construyen los partenariados y potencian los valores de participación, construcción conjunta y el empoderamiento de las personas que participan que conducen el proceso para cambiar la realidad social. Finalmente, el trabajo global de procesos (Mindell, 2014) contribuye a tomar conciencia de las trasformaciones como conjuntos de cambios en las personas y en su sistema de relaciones, incorporando los aspectos personales e interpersonales del grupo y el sistema de relaciones del municipio.

\section{INVESTIGAR A TRAVÉS DE LAS EXPERIENCIAS DE IMPULSO DEL APRENDIZAJE- SERVICIO EN EL TERRITORIO}

La investigación ha tenido como objetivos: a) impulsar procesos de implementación local del aprendizaje-servicio en diferentes territorios, acompañando a los agentes implicados en cada caso; b) analizar los aspectos clave desarrollados a través de este proceso; y c) sistematizar orientaciones y elementos a tener en

3. Con respecto a la ciudad de Barcelona, destacan el Consorcio de Educación de Barcelona, la Dirección de Justicia Global y Cooperación Internacional y el área de Políticas Alimentarias del Ayuntamiento de Barcelona; el Consejo de Innovación Pedagógica del Instituto de Educación de Barcelona y la Diputación de Barcelona. A nivel de Cataluña destaca el papel del Departamento de Educación de la Generalitat de Cataluña y los ayuntamientos de diferentes municipios, tal y como se pone de relieve en este artículo. En muchas ocasiones esta tarea se ha hecho o se está haciendo en colaboración del Centro Promotor de Aprendizaje-Servicio. 
BRENDA BÄR KWAST; LAURA CAMPO CANO Y LAURA RUBIO SERRANO LÍNEAS DE ACCIÓN Y PRINCIPIOS PARA LA INCORPORACIÓN DEL APRENDIZAJE-SERVICIO EN EL ÁMBITO LOCAL. TRABAJO EN RED EN EL TERRITORIO

cuenta para inspirar a otros territorios. En este caso, se pretende destacar aquellos aspectos a desarrollar desde el punto de vista de la persona o personas que apoyan y/o coordinan este proceso.

Para ello se ha optado por un enfoque metodológico de corte cualitativo articulado en dos aproximaciones que se centran en la investigación experiencial: por un lado a través del análisis documental de experiencias previas; y, por otro, la investigación-acción que, a través de la tarea de impulso y seguimiento en tres territorios catalanes diferentes, ha permitido explorar la realidad desde el punto de vista de quien la habita para obtener aprendizajes que orienten hacia la acción (Elliot, 1993). Sin la voluntad única de generalización, el proceso ha permitido identificar proposiciones para su comprensión, considerando la realidad temporal y espacial. Dado que las realidades están interconectadas, ello ha de permitir identificar los elementos clave, sistematizar aprendizajes y convertirlos en propuesta adaptables para otros contextos (Maykut \& Morenhouse, 1999).

La revisión sistemática ha permitido analizar experiencias anteriores ya consolidadas y previamente sistematizadas como las ocurridas en Mataró, L'Hospitalet de Llobregat, St. Vicenç dels Horts y procesos de acompañamiento con menor recorrido como Pineda, Ripollet o Santa Maria de Palau Tordera, entre otros. En este caso, el análisis ha sido posible a través de las publicaciones surgidas de la propia práctica (Batlle, 2018b), informes técnicos u otros documentos como páginas web, con la voluntad de obtener información sobre los elementos compartidos a través de los abordajes desarrollados y planificar elementos para el proceso posterior.

Por otro lado, la investigación se ha centrado en el apoyo y seguimiento, junto a los profesionales implicados, del proceso de incorporación del aprendizaje-servicio apostando por una investigación que involucra la interacción social entre investigador e informantes en el seno de estos últimos, y durante la que se recogen datos de manera sistemática y no intrusiva (Taylor \& Bodgan, 1987). En este sentido se ha tenido la oportunidad de llevar a cabo procesos de acompañamiento en tres territorios catalanes: Barcelona ciudad (a través de algunos de sus distritos), Sant Cugat y Puig-Reig.

Los territorios fueron escogidos bajo los criterios de accesibilidad, puesto que en todos ellos existía una demanda específica de la administración para introducir el aprendizaje-servicio. Los territorios mostraron interés en la propuesta y, junto con ellos, se acabó explorando las posibilidades y condiciones para fomentar buenas experiencias y garantizar una red de colaboraciones sostenible a largo plazo. También se tuvo en cuenta el criterio de diversidad, incorporando territorios de diferentes dimensiones (siguiendo la identificación que hace la Diputación de Barcelona entre municipios, ciudades pequeñas y grandes, según el volumen de población.

En los tres territorios el impulso para la implementación local del aprendizajeservicio ha supuesto un proceso de apoyo y seguimiento de dos cursos/años aproximadamente, que ha comportado el contacto periódico (mensual) con los agentes. Asimismo, también ha implicado la participación de diferentes áreas del 

EN EL ÁMBITO LOCAL. TRABAJO EN RED EN EL TERRITORIO

ayuntamiento y se ha fundamentado directamente en el trabajo junto a técnicos y técnicas del territorio. En el caso de Barcelona, cabe destacar la tarea realizada en los distritos de Sant Andreu y Sarrià-Sant Gervasi. En ambos casos, ha tenido un papel central la figura de la técnica del Área de Justicia Global y Cooperación, así como también el responsable del Centro de Recursos Pedagógicos. En el caso de Sant Cugat, la tarea se ha realizado fundamentalmente de la mano de los equipos Técnicos de Educación del ayuntamiento. Finalmente, en el caso de Puig-Reig ha habido implicación directa por parte del Concejal de Educación y la Concejala de Participación Ciudadana que, a su vez, desarrollan labores técnicas en el municipio. En este último caso, dada su dimensión, no se cuenta con equipos técnicos en todas las áreas, pero sí con una implicación elevada por parte de las personas que ocupan cargos políticos.

Los procesos realizados en cada uno de los territorios no han servido solamente para implementar el aprendizaje-servicio en cada núcleo local, sino que son la base que permite sistematizar una propuesta de líneas de acciones y principios para guiar procesos de este tipo en otros territorios. Este es precisamente el elemento central de este artículo. Cada una de estas experiencias ha permitido desarrollar acciones directas e indirectas, observaciones y sesiones de revisión y análisis respecto el proceso de implementación y arraigo del aprendizaje-servicio. Se han podido recoger notas de campo, actas de los encuentros y se ha tenido acceso y/o participación directa en la elaboración de los documentos elaborados en los territorios. A esta tarea, también hay que añadirle los espacios de contraste y reflexión con otras profesionales del grupo de investigación a lo largo de todo el periodo. De esta manera, se ha combinado la transformación del propio entorno con la generación de espacios de construcción teórico-práctica acerca de los procesos desarrollados.

\section{LÍNEAS DE ACCIÓN Y PRINCIPIOS QUE FACILITAN LA INCORPORACIÓN DEL APRENDIZAJE-SERVICIO A NIVEL TERRITORIAL}

El apoyo y estudio de las diversas experiencias ha permitido de manera progresiva, y a pesar de las diferencias entre cada uno de los núcleos territoriales, identificar y sistematizar aspectos compartidos y aprendizajes de la práctica cuya activación resultan clave para hacer crecer los proyectos de aprendizaje-servicio a nivel local y desarrollar procesos ricos de creación y arraigo de redes de colaboración y trabajo conjunto entre agentes que antes incluso no se conocían.

Entre ellos, destacan cuatro líneas de acción que, ejecutadas de manera adaptada a la realidad y el momento de cada territorio, contribuyen positivamente en el proceso de incorporación territorial del aprendizaje-servicio. La reflexión con los agentes implicados en el mismo ha permitido comprender que, a su vez, estas acciones cristalizan principios que lo orientan y dotan sentido transversalmente. Todos estos aspectos, de manera más o menos secuenciada, han de facilitar el trabajo en otros municipios y poblaciones. 
BRENDA BÄR KWAST; LAURA CAMPO CANO Y LAURA RUBIO SERRANO LÍNEAS DE ACCIÓN Y PRINCIPIOS PARA LA INCORPORACIÓN DEL APRENDIZAJE-SERVICIO EN EL ÁMBITO LOCAL. TRABAJO EN RED EN EL TERRITORIO

GRÁFICO 1

Líneas de acción y principios para la implementación territorial del Aprendizaje-Servicio

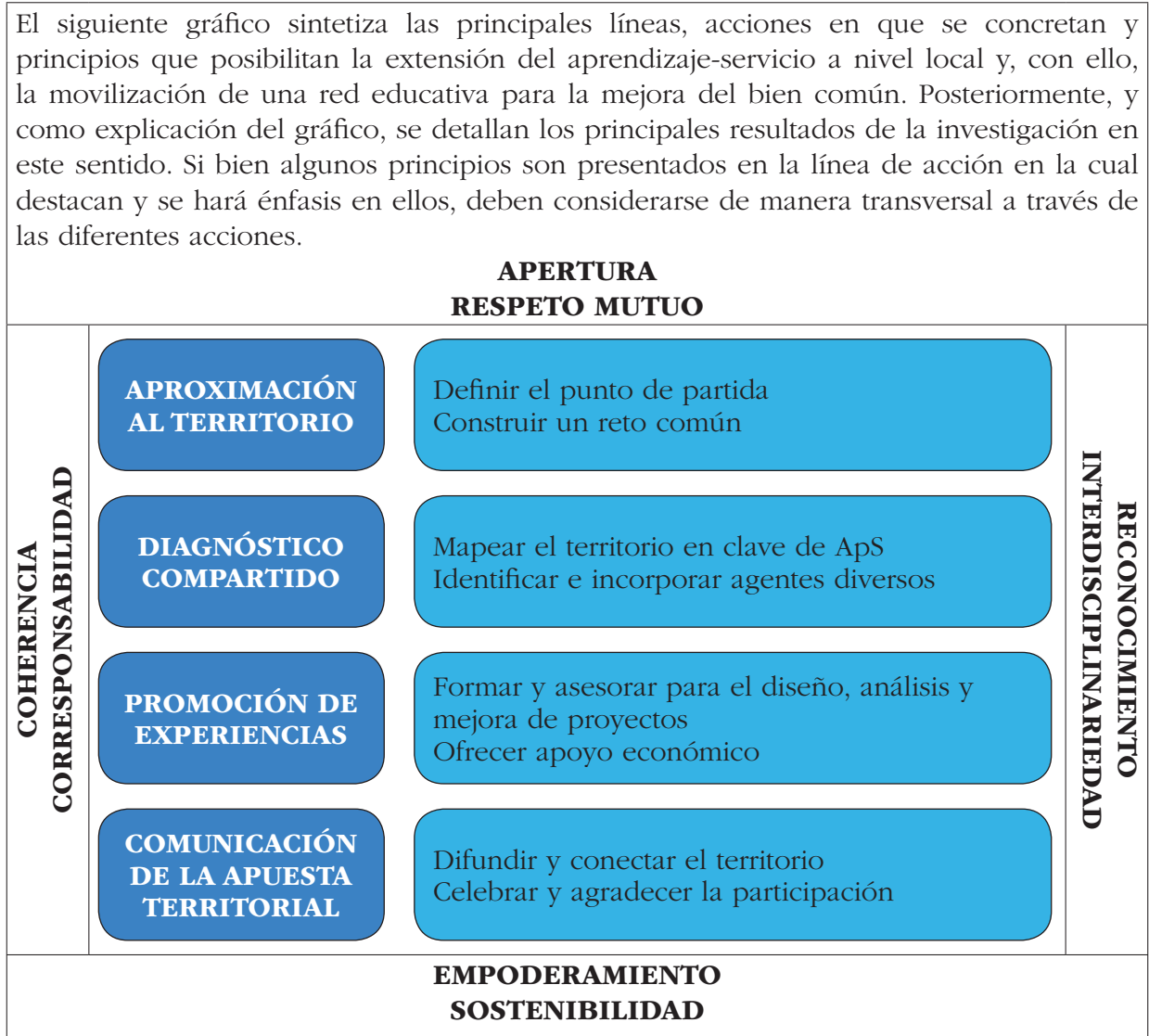

Fuente: Elaboración propia

\subsection{Aproximación al territorio}

La primera línea de acción representa el acercamiento al territorio y la toma de contacto con los agentes implicados en la incorporación del aprendizaje-servicio a nivel local en el inicio del proceso, cuando incluso ésta todavía no se visibiliza propiamente como objetivo. Su concreción supone definir el punto de partida y construir un reto común que permita caminar hacia una perspectiva territorial.

Definir el punto de partida requiere conocer la razón o razones que mueven a un territorio a iniciar este proceso. Los desencadenantes (Rueda, 1993) son pequeñas 

EN EL ÁMBITO LOCAL. TRABAJO EN RED EN EL TERRITORIO

complicaciones más o menos espontáneas y/o reflexionadas que llevan a las personas de contacto a interesarse por el aprendizaje-servicio. En los casos analizados estas figuras han sido habitualmente Técnicos de Educación, de Justicia Global y Cooperación Internacional y/o responsables de los Centros de Recursos Pedagógicos, por el hecho de tener entre sus funciones dinamizar este tipo de proyectos. Desde el primer encuentro con ellos, se han puesto de relieve desencadenantes diversos: la necesidad de buscar formación sobre metodologías más innovadoras para trabajar en centros o entidades, un encargo específico desde la dirección, la experiencia exitosa de otro municipio, una sensibilidad específica por el fuera escuela o la propia tarea de activar el Decreto de Servicio Comunitario son algunos ejemplos. Una vez identificadas estas razones resulta importante ampliar cuanto antes el círculo e identificar conjuntamente otros agentes cercanos que también puedan tener interés y capacidad de vincularse, desde sus cargos y responsabilidades, con esta propuesta pedagógica. En definitiva, a modo de ejemplo, incorporar desde este primer momento alguna otra figura técnica del ayuntamiento, el punto de información juvenil, un hotel de entidades e incluso, en los municipios o poblaciones más pequeñas, cargos políticos que combinan su tarea con otras responsabilidades y pueden implicarse directamente en el proceso.

Es en este grupo ampliado que tiene sentido construir un reto compartido que permita asumir y comprometerse con el proceso que se inicia. La construcción del reto debe nutrirse de las diferentes problemáticas que detectan los agentes, las motivaciones para mejorar en positivo y los programas o líneas de trabajo que ya están activas con las que puede vincularse el aprendizaje-servicio. Por ello resulta clave acompañar a los agentes a cuestionarse sobre qué puede aportar esta propuesta pedagógica en el municipio a nivel educativo y social y, con ello, realizar un primer análisis de necesidades del territorio. Existen núcleos territoriales que observan una problemática clara: altos índices de absentismo, centros muy estigmatizados, bajo rendimiento y, ante esta realidad, ven el aprendizaje-servicio como una propuesta interesante para dar respuesta. Otras veces la percepción es menos concreta: se piensa que las propuestas educativas que se ofrecen podrían estar más vinculadas al entorno, existe un malestar sobre la educación actual o la voluntad de generar acciones compartidas. Finalmente, también como ejemplo, existen municipios en los que el objetivo simplemente es articular experiencias de aprendizaje-servicio dispersas para darles un sentido de territorio.

Desde esta primera línea de acción y a lo largo de todo el proceso la apertura se convierte en clave para el proceso de acompañamiento del territorio para incorporar el aprendizaje-servicio. Ampliar la mirada, invitar a más agentes a participar y tener en cuenta sus ideas y preocupaciones permite captar y comprender el territorio desde nuevas perspectivas y subrayar las posibles aportaciones del aprendizaje-servicio de manera más integradora. Ello debe darse en un contexto de respeto mutuo, por lo que, desde los primeros contactos, es importante velar por un clima de escucha que se mantenga a lo largo de todo el proceso (Marchioni, 2001; Úcar y Llena, 2006). 
BRENDA BÄR KWAST; LAURA CAMPO CANO Y LAURA RUBIO SERRANO LÍNEAS DE ACCIÓN Y PRINCIPIOS PARA LA INCORPORACIÓN DEL APRENDIZAJE-SERVICIO EN EL ÁMBITO LOCAL. TRABAJO EN RED EN EL TERRITORIO

\subsection{Diagnóstico compartido}

A partir de esta primera aproximación es interesante involucrar a los agentes en un diagnóstico compartido desde la perspectiva participativa (Barbero y Cortés, 2005; Marchioni, 2001). Este permite construir una imagen de conjunto respecto las características del medio en el que se pone en marcha el proceso, delimitar las posibilidades de la apuesta y elaborar una hoja de ruta para dar respuesta a estas necesidades comunes. Para ello es necesario mapear el territorio en clave de aprendizaje-servicio e identificar nuevos agentes del mismo que puedan vincularse, de manera más o menos directa, en el desarrollo de proyectos.

Mapear el territorio en clave de aprendizaje-servicio supone explorar las experiencias e iniciativas existentes, así como los centros educativos y entidades que los promueven o podrían llegar a promoverlos. Interesa fundamentalmente identificar proyectos de aprendizaje-servicio o similares en activo, así como otras iniciativas que puedan ser repensadas y redimensionadas desde esta perspectiva comunitaria. También construir una fotografía en la que aparezcan los centros educativos y entidades sociales del contexto, como agentes susceptibles de participar en este tipo de propuestas, identificando potenciales participantes, contactos y colaboraciones previas, así como también voluntades de trabajo conjunto. Este se construye desde el conocimiento y contactos de los profesionales con las experiencias, los informes y memorias de actividades de cada curso, las propuestas presentadas a subvenciones municipales o de distrito, o la información recogida en webs de las instituciones que operan en el territorio. Teniendo en cuenta las aportaciones del mapeo colectivo y la cartografía (Ares y Risler, 2013; Lafuente y Horrillo, s.f.) su representación gráfica puede ayudar a visibilizar todos estos elementos, obtener una imagen construida desde los diferentes participantes y ofrecer datos para el análisis conjunto respecto a la tipología, ámbitos y posibilidades para activar nuevas acciones de aprendizaje-servicio.

En este mismo sentido, es necesario identificar e incorporar agentes diversos del municipio que, desde una perspectiva educativa amplia, y aprovechando las tareas que les son propias, puedan llegar a tener algún papel relevante en el desarrollo de proyectos de aprendizaje-servicio. Pueden identificarse agentes a nivel técnico local o supramunicipal, entidades de promoción educativa, equipamientos o recursos del barrio o población, o incluso figuras individuales reconocidas a nivel de territorio que pueden ser relevantes para generar o potenciar proyectos de aprendizaje-servicio desde esta perspectiva. La incorporación de estos otros agentes debe vincularse directamente a una reflexión sobre sus funciones habituales y/o sus posibles aportaciones a la apuesta de conjunto. De esta manera, se consigue que los diferentes agentes puedan encontrar su rol en el proceso, sea este el de facilitación de recursos, materiales o contactos para el desarrollo de proyectos; diseño e implementación de experiencias propias u otros aportes. En este marco, cabe destacar específicamente el interés de establecer encuentros con 

EN EL ÁMBITO LOCAL. TRABAJO EN RED EN EL TERRITORIO

técnicos/cas de las diferentes áreas del ayuntamiento para identificar necesidades sociales del territorio en las que podrían vincularse diferentes centros educativos en colaboración con la administración y entidades sociales: las dificultades para consolidar el reciclaje selectivo o promover la movilidad saludable pueden ser algunos ejemplos.

Especialmente en el diagnóstico compartido, pero también en el resto del proceso, el reconocimiento se convierte en un eje transversal para acompañar la implementación del aprendizaje-servicio en un territorio, puesto que ningún lugar parte de cero. Este principio se concreta partiendo y dando valor de las experiencias existentes o subrayando la aportación que cada agente puede hacer para contribuir en la propuesta. Dichas aportaciones exigen la interdisciplinariedad como lente para comprender el territorio y dar respuesta a sus necesidades sociales.

\subsection{Promoción de experiencias de aprendizaje-servicio}

Poner en marcha proyectos de aprendizaje-servicio con mirada de territorio requiere que todos los agentes que se van implicando progresivamente en la propuesta tengan los recursos necesarios para su correcto diseño e implementación. Desde este punto de vista, algunas de las principales acciones que cabe desarrollar en un territorio son las de formar y asesorar para la generación, implementación y evaluación de proyectos y, cuando es posible, ofrecer apoyo económico para su puesta en marcha.

Formar y asesorar para el diseño, análisis y mejora de proyectos en el territorio ha de suponer un plan formativo adaptado a cada situación y que evoluciona a lo largo del proceso. En muchas ocasiones, se considera que la formación al uso (la realización de un curso básico) ha de ser la tarea primera y más importante. Si bien esta es una acción necesaria, las diferentes experiencias muestran también que en el proceso de implementación territorial es importante no quedarse anclado en ella o en una concepción clásica de la misma y avanzar hacia propuestas diversas y ajustadas a cada situación. De esta manera, se espera que la formación específica para grupos específicos (entidades, centros o incluso familias), si es necesaria, se combine con espacios formativos que reúnan diferentes agentes de la población e incluyan la mirada territorial y movilizadora de un proceso con continuidad desde el inicio. Espacios que, en la línea del trabajo comunitario (Barbero y Cortès, 2005), inviten a participar e implicar activamente a las personas en la mejora de su propia realidad. De manera que proporcionen situaciones de encuentro para conocerse (ferias de entidades), generaren proyectos conjuntamente (talleres o laboratorios de proyectos) y reflexionen sobre la propia práctica (seminarios o grupos de trabajo). Pensar en propuestas formativas de carácter participativo, experiencial y sostenido en el tiempo es una manera de mantener viva la creación, revisión y mejora constante de los proyectos, afinando cada vez más la calidad de estos. Para ello, resulta importante facilitar testimonios de experiencias exitosas, guías metodológicas y en 
ámbitos específicos y otros recursos y herramientas que ofrecen las organizaciones que promocionan la metodología. ${ }^{4}$

Además de la formación, activar un proceso de innovación y trabajo en red requiere ofrecer apoyo económico. A pesar de no tratarse de proyectos que requieran de grandes cantidades, suele ser importante reservar una partida del presupuesto o aprovechar las oportunidades que ofrece el territorio a tal efecto. Contar con este presupuesto puede servir para hacer una convocatoria de subvenciones para el desarrollo de proyectos, poner en marcha experiencias piloto con mirada de municipio; apoyar puntualmente a las experiencias con actividades que han de enriquecer su calidad o capacidad de difusión o contribuir a la tarea que hacen las entidades promover que las entidades sociales y ONG lideren estos procesos. Algunas de estas medidas, como es el caso de las convocatorias de ayudas a proyectos del territorio, pueden convertirse en sí mismas en una fuente para conocer con más detalle los proyectos en curso en el territorio, detectar necesidades de formación y acompañamiento o, incluso, potenciar pedagógicamente la autorreflexión en el seno de centros educativos y entidades sociales a través de la formulación de propuestas.

En este contexto, cabe destacar como otro de los principios clave a lo largo de todo el proceso es el de la coherencia, que ha de permitir pasar de una apuesta en abstracto al establecimiento de espacios, momentos y recursos que posibiliten su materialización. En definitiva, ofrecer recursos específicos para que toda la comunidad pueda activar e implicarse en proyectos compartidos. Dado el contexto actual y el sentido que se pretende es desde la corresponsabilidad de las diferentes instituciones y administraciones que se puede llegar a garantizar el mejor escenario de promoción del aprendizaje-servicio en el territorio.

\subsection{Comunicación de la apuesta territorial}

Finalmente, tanto el testimonio de los participantes en las experiencias de aprendizaje-servicio; el impacto que sus proyectos van generando en el territorio o la apuesta que por el aprendizaje-servicio se está desarrollando a nivel local son aspectos de interés para ser comunicados y visibilizados con el objetivo de reconocer el trabajo hecho, inspirar nuevos proyectos y dar continuidad a la propuesta. En este sentido, son tareas importantes establecer un medio para conectar y difundir la propuesta, así como también prever espacios para celebrar y agradecer la participación de los diferentes miembros de la comunidad.

Cuando un territorio lleva a cabo un proceso de incorporación local del aprendizaje-servicio que pretende sostenerse en el tiempo necesita de vías y herramientas

4. Centro Promotor de Aprendizaje-Servicio de Catalunya (https://aprenentatgeservei.cat/), Fundazioa Zerbikas Aprendizaje y Servicio Solidario (http://www.zerbikas.es/), Centro Latinoamericano de Aprendizaje Servicio Solidario (http://www.clayss.org.ar/) y Campus Compact (https://compact.org/), entre otras. 

EN EL ÁMBITO LOCAL. TRABAJO EN RED EN EL TERRITORIO

para difundir y conectar el territorio. Con ello se busca dar a conocer las experiencias en marcha, facilitar la toma de contacto entre diferentes agentes o compartir materiales y recursos de interés para su desarrollo. Cada núcleo territorial determina aquellos formatos que le son propios y que le resultan más sencillos y eficaces para estos objetivos: catálogos o trípticos que recogen las propuestas en activo, páginas web que permiten generar alianzas entre entidades y centros educativos o programas que sintetizan la apuesta política por el aprendizaje-servicio desde una visión de conjunto. Sea cual sea el formato es importante que su misma generación ya sea útil para dinamizar el proceso, que este tenga un carácter público, que permita establecer contactos para emprender proyectos y que socialice la documentación de trabajo que se va generando y utilizando a lo largo del proceso. Además, no es suficiente con poner a punto estas herramientas, sino que también hace falta darlas a conocer, dinamizarlas para facilitar su uso.

Otra manera de dar visibilidad y sentido de conjunto del trabajo realizado en el territorio desde el aprendizaje-servicio es celebrar y agradecer la participación de los diferentes implicados en los proyectos en un marco de apuesta global. Es necesario reservar un tiempo y un espacio para valorar conjuntamente la implicación de los diferentes agentes participantes, poner de relieve la red de trabajo que se ha generado, valorar el impacto de las acciones de servicio realizado en el territorio y visibilizar la implementación del aprendizaje-servicio desde una perspectiva de conjunto. Algunos ejemplos en esta dirección son la organización de jornadas de reconocimiento de buenas prácticas en diferentes formatos, la grabación de videos que recogen la tarea realizada a lo largo del curso, el montaje de exposiciones o la publicación de artículos o entrevistas sobre las experiencias a través de la prensa u otros medios locales y las redes sociales. Estos son momentos y formas concretas para dar visibilidad y poner en valor el compromiso de los más jóvenes, valorar conjuntamente la tarea realizada o aventurar nuevos retos para continuar trabajando a través del aprendizaje-servicio.

Desde ambas acciones, se establecen buenas oportunidades para hacer patente, fortalecer o incluso buscar el compromiso (si es que anteriormente no existe) desde el punto de vista político. Si bien el aprendizaje-servicio a nivel territorial no debe de entenderse de manera partidista, este requiere inevitablemente apoyo político para su consolidación y continuidad. Favorecer que el plan de trabajo con relación al aprendizaje-servicio pase por los plenos, consejos o mesas de corresponsabilidad, así como que se explicite a través de documentos de carácter estratégico y político son algunas acciones a tener en cuenta en este sentido.

A lo largo de todo el proceso hasta aquí sistematizado acostumbra es importante explicitar un nivel cada vez mayor de empoderamiento pedagógico y organizativo por parte de los agentes y la comunidad para el desarrollo de la propuesta a nivel territorial. El trabajo global en clave de proceso (Mindell, 2014) implica introducir elementos para que las personas del territorio adquieran las habilidades necesarias para ser quienes asuman y mantengan las nuevas tareas, así como habilidades 
BRENDA BÄR KWAST; LAURA CAMPO CANO Y LAURA RUBIO SERRANO LÍNEAS DE ACCIÓN Y PRINCIPIOS PARA LA INCORPORACIÓN DEL APRENDIZAJE-SERVICIO EN EL ÁMBITO LOCAL. TRABAJO EN RED EN EL TERRITORIO

personales para facilitar las relaciones humanas que pueden condicionar el proceso. A su vez, la consolidación de este proceso debe sustentarse desde el principio de sostenibilidad, estableciendo mecanismos, dinámicas y herramientas que permitan consolidar y dar continuidad de la tarea iniciada y a las redes de trabajo generadas a este paso.

\section{A MODO DE CIERRE}

A lo largo de las páginas anteriores se han presentado procesos necesarios y maneras de activarlos que fomentan la incorporación del aprendizaje-servicio en un territorio. Se ha conseguido seguir construyendo y profundizar en temáticas abiertas en propuestas anteriores sobre la implementación del aprendizaje-servicio en el mundo local (Batlle, 2018a; Campo, et al., 2013; Puig, 2010). En esta ocasión, aportando una propuesta de sistematización de acciones construida desde diversas disciplinas comunitarias, unos principios para enforcar el trabajo y diversas reflexiones para enriquecer la relación entre aprendizaje-servicio y territorio. Estos son algunos de los resultados extraídos de la experiencia de trabajo y estudio en diferentes territorios.

A través de estos, se apunta a la generación de redes que potencian el valor de la comunidad como agente educativo en sí mismo. El artículo pretende cerrar poniendo el acento en este último aspecto a través de algunas reflexiones que, a raíz del análisis de las diferentes experiencias, han ido emergiendo como más relevantes.

En primer lugar cabe destacar que la interacción del aprendizaje-servicio con el mundo local pone de relieve su capacidad para reconocer y potenciar la agencia educativa de los diferentes agentes del territorio, más allá de los centros educativos. Durante este proceso crecen las posibilidades de colaboración, y las entidades sociales y centros educativos descubren que comparten objetivos de formación y trasformación social. De esta manera, las entidades sociales empiezan a reconocerse y a ser reconocidas, desde su rol educativo en el entorno en el que se insertan. Muchas de ellas, en ocasiones ya participaban con acciones puntuales, como charlas o talleres, pero mediante los proyectos de aprendizaje-servicio descubren un papel más activo y horizontal con los centros. Les permite visibilizarse como agentes educativos significativos de la comunidad a través de diferentes roles (Sotelino, et al., 2019), comprobando que su participación activa en los proyectos es una oportunidad para que las experiencias estén más contextualizadas a la realidad social, sean más significativas para los participantes, y, con ello, pedagógicamente más ricas.

En segundo lugar, parece de interés señalar que, a lo largo del proceso, la necesidad de colaboración en los diferentes núcleos territoriales ha supuesto la definición, creación y consolidación de grupos de impulso que han tomado formas diferentes (mesas o comisiones de impulso de aprendizaje-servicio, grupos de trabajo específicos dentro de otras estructuras como el Consejo Escolar de municipio, entre otras...). Se trata de espacios de trabajo interdisciplinar que, con una voluntad de 

EN EL ÁMBITO LOCAL. TRABAJO EN RED EN EL TERRITORIO

sumar agentes diversos y establecer circuitos de trabajo, se han articulado como motores para la implementación del aprendizaje-servicio en el territorio. No suelen constituirse desde el inicio, pero tampoco pueden ser consideradas como el resultado final del proceso de trabajo. Su configuración surge de activar mecanismos que requieran de su existencia y adaptándose a cada territorio. Estas comisiones representan un elemento clave para el trabajo en red, puesto que se convierten en un núcleo operativo necesario y realista. Pero sin duda no agotan su potencialidad, puesto que las colaboraciones que se generan en los proyectos de aprendizajeservicio son mucho más ricas y diversas.

En definitiva, el aprendizaje-servicio, desde una perspectiva local, fortalece el trabajo en red para generar territorios más educadores (Collet y Subirats, 2016). Esta propuesta invita, de manera natural, a establecer diferentes tipos de relaciones: trabajar de manera conjunta entre grupos clase, visitar una entidad cercana, salir al barrio a buscar una necesidad o realizar un servicio, o invitar a las familias a colaborar. Se trata de dinámicas que rompen los muros de los centros educativos y convierten al barrio en parte de la comunidad educativa en su conjunto. El territorio acaba siendo el espacio natural del aprendizaje-servicio porque brinda un contexto natural de necesidad, de agentes que aportan desde el rol que les es propio a cada uno de ellos y donde se potencia el capital social de los más jóvenes. Se cimientan relaciones que hacen que al papel de la escuela, de las entidades de tiempo libre u otros agentes clásicamente educativos, se le sumen otros agentes que se reconocen como educativos y con roles activos; y que todos ellos en su conjunto construyan una verdadera Ciudad Educadora. Se trata de una propuesta que potencia de manera natural que el territorio se comprometa con la educación, se fortalezca el sentimiento de pertenencia a la comunidad y una manera de entender la ciudadanía. De esta manera, toma forma la propuesta de Trilla (2005) donde las ciudades educadoras, permiten aprender "la» ciudad, «en» la ciudad y "de la" ciudad. El aprendizaje-servicio permite aprender incluso "con" y "para la» ciudad, ya que se trata de propuestas educativas que responden a necesidades de la sociedad y solo pueden desarrollarse desde ella misma.

\section{REFERENCIAS BIBLIOGRÁFICAS}

Abravanel, S. A. (2003). Building community through service-learning: the role of the community partner. Partnerships/Community, 27. https://digitalcommons.unomaha. edu/slcepartnerships/27/

Ares, P. y Risler, J. (2013). Manual de mapeo colectivo. Recursos cartográficos críticos para procesos territoriales de creación colaborativa. Tinta Limón. https://iconoclasistas.net/4322-2/

Associació Internacional de Ciutats Educadores [AICE]. (2009). Carta de Ciutats Educadores. https://www.edcities.org/ca/wp-content/uploads/sites/6/2014/03/CARTA_catala.pdf

Aznar, P. y Barrón, A. (2017). El desarrollo humano sostenible: un compromiso educativo. Teoría de la educación. Revista interuniversitaria, 29(1), 25-53. https://doi.org/10.14201/ teoredu291253 
BRENDA BÄR KWAST; LAURA CAMPO CANO Y LAURA RUBIO SERRANO LÍNEAS DE ACCIÓN Y PRINCIPIOS PARA LA INCORPORACIÓN DEL APRENDIZAJE-SERVICIO EN EL ÁMBITO LOCAL. TRABAJO EN RED EN EL TERRITORIO

Bär, B., Calvet, J., Campo, L., Puig, J. M. y Rubio, L. (2020). Consideracions i propostes per implantar l'aprenentatge servei a l'àmbit local (en prensa).

Balciuniene, I. \& Mazeikiene, N. (2008). Benefits of service-learning: Evaluations from students and communities. Social Research, 1(11), 53-66. https://www.vdu.lt/cris/ handle/20.500.12259/37663

Barbero, J. M. y Cortés, F. (2005). Trabajo Comunitario, organización y desarrollo social. Alianza Editorial.

Batlle, R. (2018a). El impulso del aprendizaje-servicio desde los ayuntamientos. Revista del Consejo Escolar del Estado, 5(8), 251-261. https://sede.educacion.gob.es/publiventa/ el-impulso-del-aprendizaje-servicio-desde-los-ayuntamientos/politica-educativa/22644

Batlle, R. (2018b). L'Hospitalet de Llobregat, modelo de generalización del aprendizaje-servicio. RIDAS, Revista Iberoamericana de Aprendizaje Servicio, 6, 54-68. https://revistes.ub.edu/ index.php/RIDAS/article/view/RIDAS2018.6.7

BIDCE - Banco Internacional de Documentos de Ciudades Educadoras (2016). Impulso del aprendizaje-servicio como herramienta de convivencia, cohesión social y participación. http://w10.bcn.es/APPS/edubidce/pubExperienciesAc.do?idexp=39085\&accio=veure\& idioma $=2$

Billig, S., Jesse, D., \& Root, S. (2005). The impact of service-learning on high school students' civic engagement. Evaluation report prepared for the Carnegie Corporation of New York. RMC Research Corporation. https://www.researchgate.net/publication/234742159_The_ Impact_of_Participation_in_Service-Learning_on_High_School_Students\%27_Civic_Engagement_CIRCLE_Working_Paper_33

Campo, L., López, A., y Puig, J. (2013). La implementació territorial de l'Aprenentatge Servei. Centre Promotor d'Aprenentatge Servei. https://aprenentatgeservei.cat/wp-content/ uploads/art_rev/Implantacio-territorial-de-lAPS.pdf

Carmona, M. y Rebollo, O. (2009). Guia operativa d'acció comunitària Acció Social i Ciutadania. Ajuntament de Barcelona. http://hdl.handle.net/11703/91427

Collet, J. y Subirats, J. (2016). Educación y territorio: 15 años de Proyectos Educativos de Ciudad (PEC) en Cataluña (España). Revista Electrónica de Geografía y Ciencias Sociales, XIX, 532. https://doi.org/10.1344/sn2016.20.15794

Dewey, J. (1916/1995). Democracia y educación. Morata.

Díaz, J. y Civís, M. (2011). Redes Socioeducativas promotoras de capital social en la comunidad: un marco teórico de referencia. Cultura y Educación: Culture and Education, 23(23), 415-429. https://doi.org/10.1174/113564011797330270

Díaz, J., Civís, M., y Longás, J. (2013). La gobernanza de redes socioeducativas: claves para una gestión exitosa. Teoría de la Educación. Revista Interuniversitaria, 35(2), 213-230. https://revistas.usal.es/index.php/1130-3743/article/view/11584/12001

Elliott, J. (1993). El cambio educativo desde la investigación-acción. Morata.

Escámez, J. (2008). Ciudadanía, Sociedad Civil y participación; una mirada pedagógica. En J. M. Touriñán (Coord.), Educación en valores, sociedad civil y desarrollo cívico (pp. 92-113). Netbiblio.

Freinet, C. (1971). Educación por el trabajo. FCE.

García Roca, J. (1994). Solidaridad y voluntariado. Al Terrae. 
BRENDA BÄR KWAST; LAURA CAMPO CANO Y LAURA RUBIO SERRANO LÍNEAS DE ACCIÓN Y PRINCIPIOS PARA LA INCORPORACIÓN DEL APRENDIZAJE-SERVICIO EN EL ÁMBITO LOCAL. TRABAJO EN RED EN EL TERRITORIO

Generalitat de Catalunya. (2015). El Servei Comunitari per a l'alumnat de secundària obligatòria. Departament d'Esenyament de la Generalitat de Catalunya. http://xtec.gencat.cat/ web/.content/comunitat/serveicomunitari/documentacio/DOCUMENT-MARC-SERVEICOMUNITARI_octubre_2015.pdf

Graell, M. y Artaraz, K. (2016). Educación y comunidad: una aproximación al partenariado educativo. Educación social: Revista de intervención socioeducativa, 64, 61-76. https:// www.raco.cat/index.php/EducacioSocial/article/view/310611 ISSN-e 2339-6954

Gordó, G. (2018). El servicio comunitario, un proyecto de innovación pedagógica. RIDAS, Revista Iberoamericana de Aprendizaje Servicio, 6, 6-18. https://doi10.1344/ridas2018.6.3

Grup de Recerca d'Educació Moral [GREM]. (2014). Rúbrica para la autoevaluación y la mejora de los proyectos de APS. Centro Promotor de Aprendizaje-Servicio. https:// aprenentatgeservei.cat/wp-content/uploads/guies/aps_autoevaluacio_cat_IMP_A5.pdf

Hannon, V., Thomas, L., Ward, S., \& Beresford, T. (2019). Local Learning Ecosystems: emerging models. WISE report series en colaboración con Inovation Unit. https://www.innovationunit.org/publications/local-learning-ecosystems-emerging-models/

Harrington, K. (2014). The impacts of service-learning on communities: Perspectives from the people. University of Georgia Theses and Dissertations. http://hdl.handle.net/10724/30462

Institut de Govern i Polítiques publiques [IGOP] - Diputació de Barcelona. (2005). Els projectes educatius de ciutat. Anàlisi de l'experiència acumulada i nova proposta metodològica. Diputació de Barcelona. https://www.diba.cat/documents/113226/127468/educacio-pdfguia_m7-pdf.pdf/0010b680-da58-4562-8cc2-49d2c224b6b6

Jacoby, B. (2003). Building partnership for Service learning. Jossey Bass.

Lafuente, A. y Horrillo, P. (s. f.). Cómo hacer un mapeo colectivo. Vivero de Iniciativas Ciudadanas. http://laaventuradeaprender.intef.es/documents/10184/67475/Como-hacer-unmapeo-colectivo

Lorenzo, M., Santos Rego, M. A., y Sotelino, A. (2015). Aprendizaje-servicio y misión cívica de la universidad. Octaedro.

Marchioni, M. (2001). Comunidad, Participación y Desarrollo. Teoría y metodología comunitaria. Editorial Popular.

Maroto, S. (2018). La educación se mueve en el territorio. Estrategias locales de aprendizajeservicio. RIDAS, Revista Iberoamericana de Aprendizaje Servicio, 6, 19-32. https://doi. org/10.1344/RIDAS2018.6.4

Martín, X. (Coord.). (2018). Educarse es de valientes. Aprendizaje-servicio con adolescentes en riesgo de exclusion social. Octaedro.

Martínez, M. (Coord.). (2010). Aprendizaje-servicio y responsabilidad social de las universidades. Octaedro y Fundació Jaume Bofill.

Martínez, M. y Puig, J. M. (2011). Aprenentatge servei: De l'Escola Nova a l'educació d'avui. Temps d'educació, 41, 11-24. http://diposit.ub.edu/dspace/bitstream/2445/113257/1/617210.pdf

Martínez Odría, A. (2003). Las actuaciones voluntarias como cauce de participación social. El interés de su inclusión en el sistema educativo formal. Estudios sobre Educación, 5, 181-190. https://dadun.unav.edu/handle/10171/8528

Maykut, P. y Morehouse, R. (1999). Investigación cualitativa. Una guía práctica y filosófica. Hurtado. 
BRENDA BÄR KWAST; LAURA CAMPO CANO Y LAURA RUBIO SERRANO LÍNEAS DE ACCIÓN Y PRINCIPIOS PARA LA INCORPORACIÓN DEL APRENDIZAJE-SERVICIO EN EL ÁMBITO LOCAL. TRABAJO EN RED EN EL TERRITORIO

Mindell, A. (2014). La democracia profunda en los foros abiertos. Pasos prácticos para la prevención y la resolución de conflictos familiares, laborales y mundiales. DDX.

Mulvihill, N., Hart, A., Northmore, S., Wolff, D., \& Pratt, J. (2011). Models of partneship working in the community-community engagement. South East Communities Dissemination Paper. http://www.coastalcommunities.org.uk/briefing\%20papers/Paper_2vgreen.pdf

National Youth Leadership Council. (2008). K-12 service-learning standards for quality practice. NYLC. https://www.nylc.org/page/standards

Naval, C., García, R., Puig, J. M., y Santos, M. A. (2011). La formación ético-cívica y el compromiso social de los estudiantes universitarios. Encounters on Education, 12, 77-91. https:// www.unav.edu/documents/29007/8315083/formacion_eticocivica.pdf

Puig, J. M. (Coord.). (2009). Aprendizaje servicio. Educación y compromiso cívico. Graó.

Puig, J. M. (Coord.). (2010). Aprenentatge Servei i Ciutadania en el marc dels Plans educatius d'entorn. Departament d'Ensenyament de la Generalitat de Catalunya. http://xtec.gencat. cat/web/.content/alfresco/d/d/workspace/SpacesStore/0044/442222967-dc67-4d73-a84307cde3cafc7a/Aprenentatge-Servei-i-PEE.pdf

Puig, J. M., Batlle, R., Bosch, C. y Palos, J. (2007). Aprendizaje-Servicio. Educar para la ciudadanía. Octaedro/ Ministerio de Educación y Ciencia. Centro de Investigación y Documentación Educativa.

Puig, J. M y Campo, L. (2012). Com impulsar l'APS a l'àmbit local? Centre Promotor d'Aprentatge Servei. https://aprenentatgeservei.cat/wp-content/uploads/guies/aps_ambit_local_web.pdf

Puig, J. M., Gijón, M., Martín, X., y Rubio, L. (2011). Aprendizaje-servicio y Educación para la ciudadanía. Revista de Educación, número extraordinario, 45-67. http://www.revistaeducacion.educacion.es/re2011/re2011_03.pdf

RMC Research Corporation. (2007). Impacts of Service-Learning on Participating K-12 Students. https://www.umassd.edu/media/umassdartmouth/leduc-center-for-civic-engagement/ service-learning-readings/sl_impacts-on-k12_students.pdf

Roldan, M., Strage, A., \& David, D. (2004). A framework for assessing academic service learning across disciplines. En M. Welch \& S.H. Billig (Ed.), New perspectives in service-learning: Research to advance the field (pp. 39-59). Information Age Publishing.

Rubio, L. y Escofet, A. (Coords.). (2017). Aprendizaje-servicio (ApS): claves para su desarrollo en la universidad. Octaedro / ICE-UB.

Rueda, J. M., (1993). Programar, implementar proyectos, evaluar. Instrumentos para la acción. Librería Certeza.

Sánchez, E., Collet, J. y Subirats, J. (Dirs.). (2009). Projectes Educatius de Ciutat: un procés permanent. Diputació de Barcelona. https://libreria.diba.cat/cat/libro/projectes-educatiusde-ciutat-un-proces-permanent-revisio-metodologica-2008_44701

Service Learning Center de la Universidad de Standford (s.f.). Community Engaged Learning. https://urbanstudies.stanford.edu/academics/community-engaged-learning

Sintes, E. (2015). Educación a tiempo completo. Aula de secundaria, 15, 26-29. https:// www-grao-com.sire.ub.edu/es/producto/la-educacion-a-tiempo-completo-as01577899

Sotelino, A., Mella, I., y Rodríguez, M. (2019). El papel de las entidades cívico-sociales en el aprendizaje-servicio. Sistematizando la participación del alumnado en el tercer sector. Teoría de la Educación. Revista Interuniversitaria, 31(2), 197-219. https://doi. org/10.14201/teri.20156 
BRENDA BÄR KWAST; LAURA CAMPO CANO Y LAURA RUBIO SERRANO LÍNEAS DE ACCIÓN Y PRINCIPIOS PARA LA INCORPORACIÓN DEL APRENDIZAJE-SERVICIO EN EL ÁMBITO LOCAL. TRABAJO EN RED EN EL TERRITORIO

Tapia, N. (2001). La solidaridad como pedagogía. El aprendizaje-servicio en la escuela. Ciudad Nueva.

Tapia, N. (2006). Aprendizaje y servicio solidario. En el sistema educativo y las organizaciones juveniles. Ciudad Nueva.

Taylor, S. J. y Bodgan, R. (1987). Introducción a los métodos cualitativos de investigación. Paidós.

Tonucci, F. (1979). La escuela como investigación. Reforma de la escuela.

Tort, A. y Collet, J. (2017). Escola, famílies i comunitat. Octaedro.

Trilla, J. (2009). El aprendizaje-servicio en la pedagogía contemporánea. En J. M. Puig (Coord.), Aprendizaje-servicio. Educación y compromiso cívico (pp. 33-52). Graó.

Trilla, J. (2005). La Ciudad educadora: Municipio y educación. En S. Peiró. (Coord.), Nuevos espacios y nuevos entornos de educación (pp. 19-42). Club Universitario.

Úcar, X. y Llena, A. (2006). Mirada y diálogos en torno a la acción comunitaria. Graó.

Winterbottom, Ch., Lake, V. E., Ethridge, E. A., Kelly, L., \& Stubblefield, J. L. (2013). Fostering social justice through Service-Learning in early childhood teacher education. Revista Internacional de Educación para la Justicia Social, 2(2), 33-53. http://hdl.handle. net/10486/660356 